\title{
Pleurectomy decortication for mesothelioma: The procedure of choice when possible
}

\author{
Raja M. Flores, MD
}

\author{
From the Department of Thoracic Surgery, Icahn School of Medicine, Mount Sinai Health System, New York, NY. \\ Disclosures: Author has nothing to disclose with regard to commercial support. \\ Received for publication Oct 9, 2015; accepted for publication Oct 13, 2015; available ahead of print \\ Nov 21, 2015 . \\ Address for reprints: Raja M. Flores, MD, Department of Thoracic Surgery, Mount Sinai Health System, Icahn \\ School of Medicine at Mount Sinai, One Gustave L. Levy Place, Box 1023 New York, NY 10029 (E-mail: \\ raja.flores@mountsinai.org). \\ J Thorac Cardiovasc Surg 2016;151:310-2 \\ $0022-5223 / \$ 36.00$ \\ Copyright $₫ 2016$ by The American Association for Thoracic Surgery \\ http://dx.doi.org/10.1016/j.jtcvs.2015.10.036
}

The art of medicine is especially invoked when a physician encounters a patient with mesothelioma, because there are an abundance of biased opinions and no standard treatment exists. The central question of surgery versus medical therapy is beyond the scope of this report. The focus will be on surgery: specifically, which surgical procedure provides optimal benefit, extrapleural pneumonectomy (EPP) or pleurectomy decortication (PD)? My stance on this subject has evolved during the past 2 decades since my days as an impressionable cardiothoracic resident with David Sugarbaker back in the mid 1990s. Then, I drank the Kool-Aid on EPP. I had no doubt as I started my thoracic career that the EPP sledgehammer was the only way to combat this dreadful disease.

Patients and surgeons would accept the increased operative risk associated with EPP if it indeed offered a real longterm survival gain. A completely empty hemithorax devoid of tumor, lung, diaphragm, and pericardium gives a nice visual that would seem ideal for the administration of highdose radiotherapy postoperatively. The real or perceived oncologic benefit of EPP was buoyed by a series of retrospective studies that demonstrated some long-term survivors, which seemed to contradict the conventional wisdom that all patients died of disease within a year of diagnosis. $^{1-7}$

Many EPP proponents believe that this procedure is theoretically a more oncologically sound operation than PD; however, the clearance of tumor from the different anatomical structures is inconsistent. The margin obtained by removal of the lung and diaphragm is not the same as the margins on the chest wall, vena cava, subclavian vessels, pericardium, aorta, esophagus, trachea, vertebral bodies, intercostal muscles, and ribs. The lung is removed because it is possible to do so, but the other structures are not removed, even though they are at the same proximity to the tumor. A true R0 resection is not possible with either EPP or PD. At best, an R1 resection may be achieved. ${ }^{8}$

The bulk of data supporting EPP are derived from a series of biased retrospective studies on subsets of highly selected

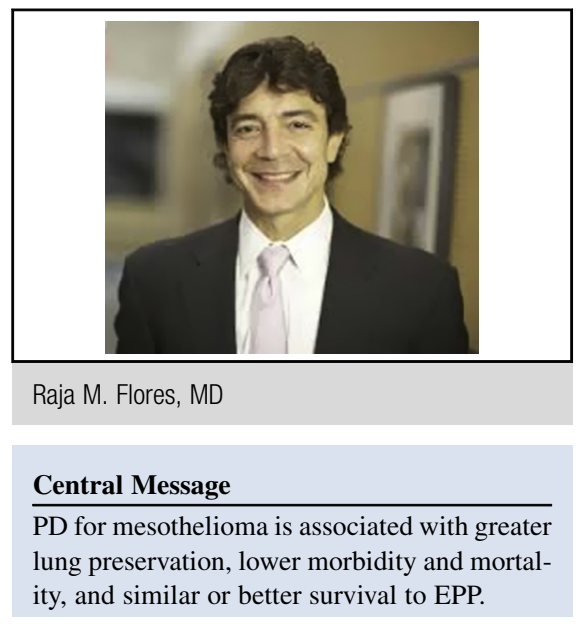

See Articles page 468 and 478.

See Editorial Commentaries page 476 and 485 .

patients. In many reports, operative deaths and patients with unresectable disease were excluded. When numbers are small, the survivor function at the far right of a KaplanMeier survival curve should be interpreted cautiously, because there are fewer patients remaining in the study group and the survival estimates are not as accurate. ${ }^{9}$ This is reflected in the observation that worsening survival is observed in almost all sequential studies when larger and larger numbers patients are added to the initial cohort. The initial studies with small sample size are always hopeful, because most patients lie to the left of the curve and a few long-term survivors give the study deceptive promise. As time progresses, these curves invariably demonstrate worse survival, as demonstrated by the most frequently cited studies. ${ }^{1-4}$ As the survival has worsened, reports have excluded biphasic and sarcomatoid histologic types to demonstrate the best survival results. ${ }^{2}$ Be wary of subsets of subsets of subsets.

The quality of the data available is poor because of the propensity of mesothelioma to infiltrate tissue planes as a sheet rather than to follow the typical TNM pattern characteristic of most solid tumors. Quality level 1 evidence is difficult to obtain, since it is difficult to stratify patients accurately by stage. Stage classification can fluctuate between stage I and III for the same patient, depending on the observer. Histologic type and N2 nodal involvement 
seem to be more accurate predictors of survival than $\mathrm{T}$ status. A valiant but failed effort was attempted by the Mesothelioma and Radical Surgery (MARS) trial. ${ }^{10}$ The only solid conclusion to be drawn from the MARS trial is that a randomized trial evaluating EPP versus nonsurgical management is not feasible. That's it! The overreaching conclusion stated in the MARS trial article that EPP is ineffective and may hurt patients was not supported by the severely underpowered data of 24 patients in the EPP arm and 26 patients in the nonsurgical arm. Is the high $18 \%$ operative mortality in the MARS trial the true operative risk of EPP? No. When patient numbers are small, the effects of selection and lead time bias can be quite large.

The lack of encouraging survival with EPP is reflected by studies on patterns of recurrence. The majority of recurrences are in the ipsilateral hemithorax. ${ }^{4,5}$ Intuitively, this would be unlikely to translate into great survival benefit. Median survival for patients treated with EPP today is no different than that for patients treated with PD in the middle of the 20th century, as reported by McCormack and colleagues. ${ }^{6}$ We have come full circle.

A combination of the best available data and common sense can go a long way. So we are left with the vast majority of data being biased by surgical treatment, which is why untreated patients with mesothelioma from large databases, such as the Surveillance, Epidemiology, and End Results database, provide valuable information with regard to the natural history. ${ }^{7}$ Does surgery really result in better survival, or is this just a reflection of those who have better performance status and smaller disease burden leading to better survival regardless of treatment? In light of this question, when a 5-year survival of $15 \%$ is observed in patients without surgical resection, this should be the absolute

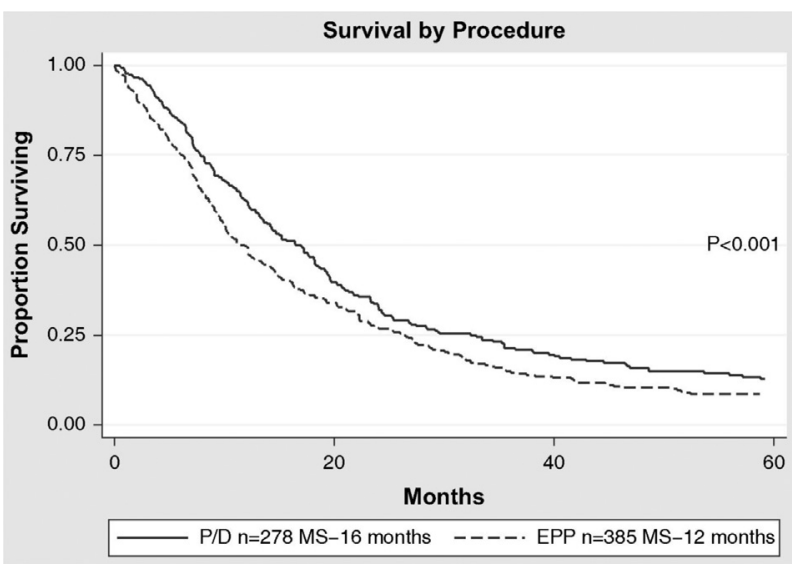

FIGURE 1. Kaplan-Meier curve for extrapleural pneumonectomy (EPP) versus pleurectomy decortication $(P / D)$. (Used with permission from: Flores RM, Pass HI, Seshan VE, Dycoco J, Zakowski M, Carbone M, et al. Extrapleural pneumonectomy vs pleurectomy/decortication in the surgical management of malignant pleural mesothelioma: results in 663 patients. J Thorac Cardiovasc Surg. 2008;135:620-6.)
TABLE 1. Multivariate model comparing 663 patients after either extrapleural pneumonectomy $(\mathbf{n}=\mathbf{3 8 5})$ or pleurectomy decortication $(\mathbf{n}=\mathbf{2 7 8})$

\begin{tabular}{llcc}
\hline & HR & CI & $\boldsymbol{P}$ value \\
\hline Age & 1.0 & $1.01-1.02$ & $<.001$ \\
Male sex & 1.3 & $1.05-1.64$ & .02 \\
EPP & 1.4 & $1.18-1.69$ & $<.001$ \\
Nonepithelioid & 1.3 & $1.11-1.60$ & $<.001$ \\
Stage III/IV & 1.4 & $1.28-1.55$ & $<.001$ \\
Multimodality therapy & 0.45 & $0.38-0.54$ & $<.001$ \\
\hline
\end{tabular}

$H R$, Hazard ratio; $C I, 95 \%$ confidence interval; $E P P$, extrapleural pneumonectomy.

minimum standard against which all other treatments should be compared.

The only large head-to-head comparison of EPP versus PD by experienced mesothelioma surgeons demonstrated better survival associated with PD than with EPP after controlling for histologic type, stage, multimodality treatment, and sex. ${ }^{7}$ (Figure 1 and Table 1.) Although arguments may be made because of the retrospective nature of this study, surgeon selection bias, and the variations in adjuvant treatment, the surgical numbers are sizeable for comparison. If we want to use EPP despite its higher operative risk, this would make sense if we saw huge differences in survival, but such differences are not observed. We actually see a worse survival than with PD. Practically, PD preserves more lung and demonstrates (at least) similar or better overall survival and decreased postoperative morbidity and mortality when compared with EPP, while patterns of recurrence remain local. The perceived oncologic benefits of EPP do not appear to translate into real benefits for the patient.

In conclusion, PD appears to provide the most benefit and the least harm to the patient with mesothelioma undergoing surgery. Many cases can be performed while sparing the underlying lung. In some cases, no surgical procedure will rid the patient of all gross disease regardless of lung removal. The real question arises in the few cases in which removal of all gross tumor requires the resection of the underlying lung with an EPP. The best available data sway me away from EPP, but I am not ready to abandon it completely. When faced with such situations, it is critical to know your patient and engage the patient and the family in treatment decisions. Herein lies the art of medicine-and surgery

\section{References}

1. Sugarbaker DJ, Strauss GM, Lynch TJ, Richards W, Mentzer SJ, Lee TH, et al. Node status has prognostic significance in the multimodality therapy of diffuse, malignant mesothelioma. J Clin Oncol. 1993;11:1172-8.

2. Sugarbaker DJ, Richards WG, Bueno R. Extrapleural pneumonectomy in the treatment of epithelioid malignant pleural mesothelioma: novel prognostic implications of combined N1 and N2 nodal involvement based on experience in 529 patients. Ann Surg. 2014;260:577-80; discussion 580-2.

3. Sugarbaker DJ, Flores RM, Jaklitsch MT, Richards WG, Strauss GM, Corson JM, et al. Resection margins, extrapleural nodal status, and cell type determine postoperative long-term survival in trimodality therapy of malignant 
pleural mesothelioma: results in 183 patients. J Thorac Cardiovasc Surg. 1999; 117:54-63; discussion 63-5.

4. Baldini EH, Recht A, Strauss GM, DeCamp MM Jr, Swanson SJ, Liptay MJ, et al. Patterns of failure after trimodality therapy for malignant pleural mesothelioma. Ann Thorac Surg. 1997;63:334-8.

5. Flores RM, Pass HI, Seshan VE, Dycoco J, Zakowski M, Carbone M, et al. Extrapleural pneumonectomy versus pleurectomy/decortication in the surgical management of malignant pleural mesothelioma: results in 663 patients. J Thorac Cardiovasc Surg. 2008;135:620-6.

6. McCormack PM, Nagasaki F, Hilaris BS, Martini N. Surgical treatment of pleural mesothelioma. J Thorac Cardiovasc Surg. 1982;84:834-42.

7. Flores RM, Riedel E, Donington JS, Alago W, Ihekweazu U, Krug L, et al. Frequency of use and predictors of cancer directed surgery in the management of malignant pleural mesothelioma in a community-based (Surveillance, Epidemiology, and End Results [SEER]) population. J Thorac Oncol. 2010;5:1649-54.

8. Cameron RB. Extrapleural pneumonectomy is the preferred surgical management in the therapy of pleural mesothelioma: con argument. Ann Surg Oncol. 2007; 14:1249-53.

9. Rich JT, Neely JG, Paniello RC, Voelker CC, Nussenbaum B, Wang EW. A practical guide to understanding Kaplan-Meier curves. Otolaryngol Head Neck Surg. 2010;143:331-6.

10. Treasure T, Lang-Lazdunski L, Waller D, Bliss JM, Tan C, Entwisle J, et al; MARS trialists. Extra-pleural pneumonectomy versus no extra-pleural pneumonectomy for patients with malignant pleural mesothelioma: clinical outcomes of the Mesothelioma and Radical Surgery (MARS) randomised feasibility study. Lancet Oncol. 2011;12:763-72. 